eating and body image were rarely discussed. 118 referrals were made as a result of HEADSS assessments; which constituted $54 \%$ of all encounters in which at least 1 category was screened. Referrals were limited in their scope, the majority being made to Social Services and safeguarding; smaller numbers were made to CAMHS, Red Thread and local Sexual Health services.

Conclusion The use of HEADSS psychosocial screening, which was largely performed by doctors, was inadequate amongst the 13 to 20 year age group. The high rate of referral when HEADSS screening was performed suggests it is of value. This data also suggests a need for multidisciplinary education around HEADSS screening, and exploration of referral pathways to other services that address the needs of young people presenting to the Emergency Department.

\section{P9 GETTING IT RIGHT FOR ADOLESCENTS: WHAT DO THEY WANT? PATIENT EXPERIENCE OF A HOSPITAL STAY}

${ }^{1,2} \mathrm{~K}$ Vutipongsatorn*, ${ }^{1,2} \mathrm{~V}$ Ramadoss, ${ }^{2} \mathrm{D}$ Preston, ${ }^{2} \mathrm{~K}$ Malbon. 'Department of Medicine, Imperial College London, London, UK; ${ }^{2}$ Department of Paediatrics, St Mary's Hospital, London, UK

\subsection{6/bmjpo-2019-RCPCH-SAHM.17}

Aims To assess the experiences of adolescent patients in an inpatient setting at a tertiary hospital.

Methods From October 2018 to March 2019, patients aged 13-23 years old were identified using the electronic record system and were approached to complete an anonymous 29question survey. The survey asked participants about their well-being, appropriateness of environment and awareness of adolescence-related services.

Results 30 patients completed the survey. 48\% were under sixteen years old while $41 \%$ were sixteen to twenty-one. Approximately half (47\%) were male. $63 \%$ and $37 \%$ were admitted to the paediatric and adult wards respectively. 90\% felt this was appropriate. Among the 10\% who indicated otherwise, reasons given were 'being frightened' by older patients and 'sharing toilets'. The majority (93\%) felt that they understood their condition. However, 83\% reported that they had a say in management with 53\% feeling that their parents did most of the talking during consultations. The most common topics that participants would like to discuss were education (43\%), mental health (29\%) and weight management (24\%). 28\% suffered from low mood or felt the need to seek mental health support. $41 \%$ did not know where to access support for sexual health and contraception. $32 \%$ had discussions about alcohol, smoking or drugs. Among these, only 38\% were told about options for cessation and further help. Three themes were identified as suggested areas of improvement. 1. Participants would like improved facilities with individual toilets, study rooms and private space for adolescents. 2. More entertainment options such as game consoles and better Internet access were proposed. 3. Patients felt they would benefit from more nurses and a quicker discharge process.

Conclusion Adolescents should be placed in more appropriate environments when admitted to hospital to maximise the comfort of their stay, co-horting patients with those of a similar age and providing young person friendly entertainment and resource. Additionally, professionals should opportunistically provide education about their physical and mental health and emotional wellbeing. Finally, referral pathways to community partners need to be established to aid discharge and provide support once a young person has left the hospital.

\section{P10 FEASIBILITY, ACCEPTABILITY AND EFFECTIVENESS OF YOUNG-PEOPLE SPECIFIC, INTEGRATED OUT-OF- HOSPITAL SERVICES: A SYSTEMATIC REVIEW}

${ }^{1,2}$ A Pandey* ${ }^{*}$ R Viner, ${ }^{1}$ A Gireesh. ${ }^{1}$ Population, Policy and Practice Programme, UCL GOS Institute of Child Health, London, UK; ${ }^{2}$ National Guideline Alliance, Royal College of Obstetricians and Gynaecologists, London, UK

\subsection{6/bmjpo-2019-RCPCH-SAHM. 18}

Aims Although there is a broad agreement on the need of integrated care in young people, there has been no systematic effort to evaluate the provision of integrated out of hospital health services for this group. The objective of the review was to examine the effectiveness, feasibility, and acceptability of young-people specific integrated out-of-hospital services.

Methods We systematically searched for relevant peer-reviewed articles published before 2018 using an iterative search strategy: Medline, EMBASE/Ovid, CINAHL Plus and CENTRAL. In addition, a hand search of reference lists and relevant topics from systematic reviews and grey literature were also performed. Data were extracted and synthesised across these three themes: (i) effectiveness, (ii) feasibility and (iii) acceptability of integrated out-of-hospital health services for young people (10-24 years).

Results Following title and abstract screening, 177 full text articles were reviewed and 31 studies meeting the inclusion criteria were included and summarized in a narrative review. Services offered varied across different studies. Fourteen services focused on mental health services. Other services either had a holistic focus based within primary care, provided interdisciplinary care, HIV and pregnancy prevention services or reports were unclear in their descriptions of the service focus. The most common intervention described was individual counselling. Services were generally acceptable and effective, though a small number also experienced no change or a decline in their condition. Common features of effective and acceptable programs were being youth-friendly (staff and environment) and welcoming, being staffed by young people, maintaining confidentiality and privacy, wide range of integrated services available in one place, convenient location, community-based outreach services, disease-oriented care facilitation, and integration within existing services, nurse/peer-led care, consistent service and collaborative governance. However inconsistency in findings was observed and quality of evidence was weak.

Conclusion There is some preliminary evidence on the effectiveness and acceptability of out-of-hospital integrated youth services, but, limited evidence to support the feasibility. Further research is required to evaluate the strength of such youth-focussed services in order to maximise patient benefit and to inform public policy. Considering the limitations in current evidence base including lack of direct comparison of services; large comparative studies controlling for possible confounders is merited. 